\title{
Approach to drug discovery integrating large-scale medical information databases with clinical and basic research
}

\author{
Mitsuhiro Goda ${ }^{1}$, Yoshito Zamami ${ }^{1,2}$, Takahiro Niimura $^{2}$, Kenshi Takechi $^{3}$, Masayuki Chuma ${ }^{3}$, \\ Yuki Izawa-Ishizawa ${ }^{4}$, Keisuke Ishizawa ${ }^{1,2}$ \\ ${ }^{I}$ Dept. Pharm., Tokushima Univ. Hosp., ${ }^{2}$ Dept. Clin. Pharmacol. Ther., Grad. Sch. Biomed. Sci., Tokushima Univ., \\ ${ }^{3}$ Clin. Trial Ctr. Developmental Therap., Tokushima Univ. Hosp., ${ }^{4}$ AWA Support Ctr, Tokushima Univ.
}

In recent years, large-scale medical information databases for diseases and their related side effects have been used to accurately assess the effects and side effects of clinically used drugs. Integration of basic and clinical research with medical information databases can enable various analyses based on real-world data. We used this approach to improve the quality of cancer treatment by developing drugs to reduce the side effects caused by anticancer drugs. Focusing on acute kidney injury (AKI) caused by cisplatin (CDDP), we found that several existing drugs suppress the occurrence of CDDP-induced AKI in the FAERS (FDA Adverse Event Reporting System). In vitro and in vivo experiments revealed that administration of candidate drugs extracted by FAERS analysis significantly suppressed CDDP-induced AKI. These results suggest that the existing pharmaceutical products selected using FAERS could prevent CDDP-induced AKI. Effective use of medical data is a major cornerstone for drug discovery and improvement of the quality of life for patients with cancer. This novel strategy will provide a means to expand the possibilities of drug delivery and to elucidate mechanisms of unknown diseases. 